\title{
Circulating linoleic acid and alpha-linolenic acid and glucose metabolism: the Hoorn Study
}

\author{
Mieke Cabout ${ }^{1,2} \cdot$ Marjan Alssema $^{2,3} \cdot$ Giel Nijpels $^{4}$ - Coen D. A. Stehouwer ${ }^{5}$. \\ Peter L. Zock ${ }^{2}$ - Ingeborg A. Brouwer ${ }^{1}$ Amany K. Elshorbagy ${ }^{6}$ Helga Refsum ${ }^{6,7}$. \\ Jacqueline M. Dekker ${ }^{3}$
}

Received: 16 February 2016 / Accepted: 28 June 2016 / Published online: 14 July 2016

(C) The Author(s) 2016. This article is published with open access at Springerlink.com

\begin{abstract}
Purpose Data on the relation between linoleic acid (LA) and alpha-linolenic acid (ALA) and type 2 diabetes mellitus (T2DM) risk are scarce and inconsistent. The aim of this study was to investigate the association of serum LA and ALA with fasting and $2 \mathrm{~h}$ post-load plasma glucose and glycated hemoglobin (HbA1c).

Method This study included 667 participants from third examination (2000) of the population-based Hoorn study in which individuals with glucose intolerance were overrepresented. Fatty acid profiles in serum total lipids were measured at baseline, in 2000. Diabetes risk markers were measured at baseline and follow-up in 2008. Linear regression models were used in cross-sectional and prospective analyses.

Results In cross-sectional analyses $(n=667)$, serum LA was inversely associated with plasma glucose, both in
\end{abstract}

Electronic supplementary material The online version of this article (doi:10.1007/s00394-016-1261-6) contains supplementary material, which is available to authorized users.

Mieke Cabout

mieke.cabout@vu.nl

1 Department of Health Sciences and EMGO Institute for Health and Care Research, Vrije Universiteit Amsterdam, Amsterdam, The Netherlands

2 Unilever Research and Development, Vlaardingen, The Netherlands

3 Department of Epidemiology and Biostatistics and EMGO Institute for Health and Care Research, VU University Medical Center, Amsterdam, The Netherlands

4 Department of General Practice and EMGO Institute for Health and Care Research, VU University Medical Center, Amsterdam, The Netherlands fasting conditions $(B=-0.024[-0.045,-0.002])$ and $2 \mathrm{~h}$ after glucose tolerance test $(B=-0.099[-0.158$, $-0.039])$, but not with HbAlc $(B=0.000$ [ -0.014 , $0.013]$ ), after adjustment for relevant factors. In prospective analyses $(n=257)$, serum LA was not associated with fasting $(B=0.003[-0.019,0.025])$ or post-load glucose $(B=-0.026[-0.100,0.049])$. Furthermore, no significant associations were found between serum ALA and glucose metabolism in cross-sectional or prospective analyses.

Conclusions In this study, serum LA was inversely associated with fasting and post-load glucose in cross-sectional, but not in prospective analyses. Further studies are needed to elucidate the exact role of serum LA and ALA levels and dietary polyunsaturated fatty acids in glucose metabolism.

Keywords Serum fatty acids $\cdot$ Linoleic acid · Alpha-linolenic acid · Type 2 diabetes · Glucose · Hemoglobin A1c

5 Department of Internal Medicine and Cardiovascular Research Institute Maastricht (CARIM), Maastricht University Medical Centre, Maastricht, The Netherlands

6 Institute of Basic Medical Sciences, Department of Nutrition, University of Oslo, Oslo, Norway

7 Department of Pharmacology, University of Oxford, Oxford, UK 


\section{Introduction}

Dietary fatty acids affect blood lipid concentrations [1] and are related to the risk of developing coronary heart disease (CHD) [2]. Therefore, most nutrition recommendations advise to limit intakes of saturated fatty acids (SFA) and trans fats and replace these with unsaturated fatty acids, in particular polyunsaturated fatty acids (PUFA) for optimal CHD risk reduction, which in the diet mainly occur as omega-6 linoleic acid (LA) and omega- 3 alpha-linoleic acid (ALA) [3-5].

Type 2 diabetes mellitus (T2DM) is a rapidly growing chronic metabolic disease [6] that not only confers a high risk of complications, but also puts a heavy burden on patients' quality of life and on healthcare resources. Data on the role of dietary fatty acid intake, in particular on the plant-based PUFA linoleic acid (LA) and alpha-linolenic acid (ALA), in the development of T2DM are limited. According to FAO/WHO guidelines, there is probable but inconclusive evidence that increasing LA lowers the risk of developing T2DM [7]. Some prospective cohorts reported that higher dietary LA intake is associated with lower T2DM risk [8-10], while others showed no significant associations [11-13] or even a positive association [14].

Dietary intake is often difficult to determine due to the limited accuracy of the measurement and the food composition tables, and the tendency to underestimate total energy intake including fat intake $[15,16]$. The intake of the nonendogenously produced fatty acids LA and ALA can also be assessed more objectively via measurement of circulating fatty acids in serum, plasma or erythrocytes. These levels provide a relatively accurate reflection of the dietary fatty acid intake [17] over past several weeks or months [18]. The association of fatty acids in serum, but more often in plasma or erythrocytes, with T2DM has been investigated in a few prospective studies. Most researchers observed that higher proportions of LA were associated with a significantly lower T2DM risk [11, 13, 19-22], but others found no association [23]. A recent systematic review on the omega-3 fatty acids ALA, eicosapentaenoic acid (EPA), and docosahexaenoic acid (DHA), including six cohorts, concluded that higher circulating ALA and not EPA or DHA was associated with a nonsignificant trend toward lower risk of T2DM [24].

Researchers that studied both the fatty acid intake from diet and fatty acids in the circulation concluded that associations with diabetes risk were stronger with the use of circulating LA and ALA blood levels than with dietary intakes assessed by food frequency questionnaire (FFQ) [11, 13, 14]. Most earlier studies on these fatty acids and diabetes incidence relied on diagnosed, self-reported diabetes which is an underestimation of the total burden of diabetes. It is estimated that worldwide, half of the diabetes patients are undiagnosed [6]. In the present study, all current markers used to diagnose T2DM were measured [11]. In this data set, we investigated the cross-sectional and the prospective associations of serum LA or ALA with fasting plasma glucose (FPG), post-load glucose (PLG), and glycated hemoglobin (HbA1c).

\section{Method}

\section{Study population}

The Hoorn study is an observational cohort study on glucose metabolism in the general Dutch Caucasian population which included 2484 participants, aged 50-74 years at study initiation in 1989. The detailed research design and methods of this initial Hoorn Study are described elsewhere [25]. For the examination cycle in 2000-2001, an invitation was sent to all surviving patients with T2DM $(n=176)$ and random samples of individuals with impaired glucose tolerance $(n=193)$ and normal glucose tolerance $(n=705)$, based on their glucose tolerance status at the 1996-1998 examination. Of all invited participants, $648(60 \%)$ participated in this study [26]. In addition, 195 (90\%) of the 217 newly diagnosed patients with T2DM from Hoorn screening study (1998-2000) were included, as well as 60 diabetes patients, newly diagnosed in general practice [27]. We excluded people taking oral glucose lowering medication $(n=48)$, patients with known diabetes at previous Hoorn study examinations $(n=19)$, people with missing information on diabetes status $(n=15)$, and people with missing information on serum fatty acids $(n=154)$. In total, 667 individuals were included in the current cross-sectional analyses. Of the $n=667$ participants at baseline, $n=417$ participated in the follow-up examination performed in 2008, where they underwent an interview to obtain general and medical history-related information, a physical examination, and analysis of blood samples [28]. For the prospective analysis, we additionally excluded all baseline T2DM patients $(n=160)$ because treatment initiation may have impacted on the outcomes. The 257 individuals were included in the current prospective analyses. This study was conducted according to the guidelines laid down in the Declaration of Helsinki, and all procedures involving human participants were approved by the VU University Medical Centre Ethics Committee. Written informed consent was obtained from all participants.

\section{Fatty acid profile}

A fasting blood sample was drawn for the determination of serum total lipids at baseline. The VU Medical Center stored the serum samples for $\sim 10$ years at $-80{ }^{\circ} \mathrm{C}$. Fatty 
acid profiles in serum total lipids were assayed by gas liquid chromatography with flame ionization detection at AS Vitas, Oslo Innovation Center, Oslo, Norway. Serum samples, thawed in fridge overnight, were vortexed, centrifuged, and transferred into new vials. Internal standard (triheptadecanoin) was added, and fatty acids were methylated into methyl esters (FAMEs) with $\mathrm{MeOH} \mathrm{HCl}$. The FAMEs were extracted with hexane. After neutralization with $\mathrm{KOH}$ in water, mixing and centrifuging, the hexane phase was injected into the GC-FID. Analysis was performed on an Agilent 7890A GC with a splitless injector, a 7683B automatic liquid sampler, and flame ionization detection (Agilent Technologies, Palo Alto, CA, USA). Separations were obtained using a SP-2380 column $(30 \mathrm{~m} \times 0.25 \mathrm{~mm}$ i.d. $\times 0.25 \mu \mathrm{m}$ film thickness) from Supelco (Bellafonte, PA, USA). FAMEs were identified by comparison with known standards. An external standard containing known amounts of relevant FAMEs (Supelco 37 component FAME Mix) was included in each run to correct for differences in fatty acid response factors. Fatty acid content was calculated based on the area $\%$ of peaks and response factors relative to 18:0. The method has been validated for quantification of fatty acids according to US FDA Guidance Document on Bioanalytical Method Validation. The inter-assay coefficient of variation varied between 2 and $4 \%$.

\section{Glucose levels}

FPG levels were determined in serum by a hexokinase method (Roche Diagnostics GmbH, Mannheim, Germany), and samples for PLG were taken $2 \mathrm{~h}$ after ingestion of a standard 75-g oral glucose tolerance test at both examinations. The HbAlc result at baseline was calculated as a ratio to hemoglobin by an ion-exchange high-performance liquid chromatography (HPLC) based on the separation on a Mono-S column (Pharmacia, Uppsala, Sweden). At follow-up $\mathrm{HbAlc}$ was measured by a turbidimetric inhibition immunoassay. The Diabetes Control and Complications Trial (DCCT) standardization of $\mathrm{HbAlc}$ was only present in the follow-up measurements, so an internal validation between HbA1c measurements and DCCT standardization methods was needed. Conformity between the two HbAlc measuring methods was found that disputes a significant impact of the dissimilar laboratory methods on our outcomes [29, 30]. Glucose tolerance status at baseline was determined according to the most recent WHO criteria $[31,32]$. FPG $(\geq 7.0 \mathrm{mmol} / \mathrm{L})$, PLG $(\geq 11.1 \mathrm{mmol} / \mathrm{L})$, and HbAlc $(\geq 6.5 \%)$ were used as the cutoff value for classifying T2DM in this study.

\section{Other measurements}

BMI and waist-hip ratio (WHR) were assessed at baseline and follow-up using a standardized protocol as described elsewhere [33]. We collected self-reported information on medical history, family history in diabetes, medication use and lifestyle factors including physical activity [34]. Participants completed a validated semiquantitative FFQ [35] about their diet to assess average food intakes. Dietary intakes of total energy, total fatty acids, and specific fatty acids (En \%) were calculated using an extended version of the Dutch Food Composition table from 2001 (NEVO) [36]. Enzymatic methods (Roche, Mannheim, Germany) were used to measure triglycerides and cholesterol values.

\section{Statistical analysis}

Baseline characteristics of participants were presented in quartiles according to serum proportion of LA and ALA. To investigate the linear trend between serum LA or ALA and all baseline characteristics, we utilized a crude regression analysis with continuous measures.

After checking the data for potential effect modification by age, sex, use of statins, T2DM, or cohort origin (Hoorn study vs. Hoorn screening study), multiple linear regression tests were performed to investigate the cross-sectional association between baseline serum LA or ALA and baseline glucose parameters (FPG, PLG, and $\mathrm{HbA1c}$ ). Adjustments were added in a stepwise approach, with Model III being the fully adjusted model that included adjustments for sex, age, total energy intake (kcal), BMI $\left(\mathrm{kg} / \mathrm{m}^{2}\right)$ and WHR (waist/hip ratio), physical activity (PA)(min/week), fiber $(\mathrm{g} / \mathrm{d})$, total dietary SFA $(E \%)$, alcohol $(E \%)$ and level of education. Additional confounding or mediation was addressed by stepwise adding other risk factors for T2DM: total cholesterol $(\mathrm{mmol} / \mathrm{L})$, use of statins (yes/no), or triglycerides $(\mathrm{mmol} / \mathrm{L})$.

Multiple linear regression tests were performed to investigate the association between serum LA and ALA proportions at baseline and FPG, PLG, and HbA1c at follow-up. We adjusted for the baseline levels of FPG, PLG, or HbA1c as well as for sex, age, total energy intake, and other risk factors similar to the models used in the cross-sectional analyses.

In addition to data on serum fatty acids proportions (as $\%$ of total fatty acids), absolute levels of serum fatty acids and data on dietary intake of LA and ALA via a FFQ were also available. To examine the validity of serum fatty acid proportions as markers of fatty acid intake, the Pearson correlation coefficients of total dietary LA and ALA with serum proportions and with absolute levels of LA or ALA were calculated. The associations between dietary intake of LA and ALA and the three blood glucose parameters were assessed with linear regression analyses.

SPSS version 18.0 was used for the statistical analyses (SPSS Inc. Released 2009. PASW Statistics for Windows, version 18.0. Chicago: SPSS Inc). A $p$ value below 0.05 
was considered statistically significant, except for interaction terms, where we used $p<0.10$.

\section{Results}

\section{Baseline characteristics}

The mean age of the participants was 69 years (SD 7 years), and $49 \%$ was male. In total, $128(28 \%)$ of the 667 study participants were newly diagnosed with T2DM. Mean proportions of serum LA and ALA were $26.98 \% \pm 3.81$ and $0.53 \% \pm 0.15$. The complete fatty acid profile of the population is shown in Supplementary Table 1. Characteristics of the study subjects across serum LA and ALA quartiles are displayed in Table 1. Individuals with higher serum LA proportions were more frequently male and had significantly lower BMI, WHR, dietary intake of SFA, triglycerides level, statins use and lower proportions of impaired fasting glucose (IFG)/impaired glucose tolerance (IGT), and T2DM. Also their levels of physical activity, LDL and HDL cholesterol levels were significantly higher, as well as their dietary intake of LA and ALA (\%) and dietary fiber. Individuals with higher serum ALA proportions had significantly higher levels of physical activity and triglycerides, higher dietary ALA and fiber intake, and lower levels of HDL cholesterol.

Mean levels of LA were associated with glucose tolerance state [27.7 \% (3.6) for NGM, $26.5 \%$ (3.8) for IFG/ IGT, and $26.4 \%$ (4.0) for T2DM, ANOVA $p$ value $<0.05$ ]. Mean levels of ALA were not associated with glucose tolerance state $[0.54 \%(0.15)$ for NGM, $0.53 \%(0.14)$ for IFG/IGT, and $0.52 \%(0.17)$ for T2DM, ANOVA not significant].

\section{Cross-sectional analyses}

Baseline serum LA proportions were inversely and significantly associated with FPG $(B=-0.024[-0.045$, $-0.002])$ and PLG $(B=-0.099[-0.158,-0.039])$ in the fully adjusted model. For PLG, this result remained significant after further adjustment for serum cholesterol and use of statins $(B=-0.096[-0.156,-0.035])$ but not triglycerides. Significance was lost for FPG when additionally adjusted for cholesterol and statin use and triglycerides. There was no significant association between baseline serum LA proportions and baseline HbA1c (see Table 2). There was no significant association between serum ALA proportions and any of the three baseline blood glucose parameters. However, adjustment for triglycerides resulted in an inverse significant association of serum ALA with FPG $(B=-0.810[-1.333,-0.288])$ and PLG $(B=-2.335[-3.837,-0.834])$. There were no significant interactions between serum levels of LA or ALA and age, sex, use of statins, cohort origin, and T2DM $(p>0.10)$, except for the association between serum ALA and FPG, which was in the fully adjusted model stronger among subjects not taking statins ( $p$ value interaction $=0.052$ ). Associations of serum polyunsaturated fatty acid levels other than LA and ALA are presented in Supplementary Table 2.

\section{Prospective analyses}

After a mean follow-up time of $7.44 \pm 0.55$ year, 39 patients (20 male) out of 257 had developed T2DM. As shown in Table 3, baseline serum LA proportions were inversely and statistically significantly associated PLG $(B=-0.112[-0.187,-0.037])$ at follow-up in Model I. However, this association was no longer statistically significant after adjustments for baseline PLG, and other covariates $B=-0.026$ [ $-0.100,0.015]$, respectively). There was no association between baseline serum LA proportions and FPG or HbA1c levels at follow-up. Baseline serum ALA proportions were not significantly associated with FPG, PLG or HbA1c.

\section{Additional analyses}

There was a significant correlation between dietary LA intake $(E \%)$ and serum proportions (\% of total fatty acids) of LA $(r=0.343, p<0.001)$ and a modest correlation with concentrations (micrograms $/ \mathrm{ml})$ of LA $(r=0.218$, $p<0.001)$. There was a weaker correlation between dietary ALA intake and serum proportions of ALA $(r=0.134$, $p<0.001$ ) or concentrations (micrograms $/ \mathrm{ml}$ ) of ALA $(r=0.128, p<0.001)$. Dietary LA intake was not significantly associated with any of the blood glucose parameters $(B=0.021[-0.025,0.068]$ for FPG $B=0.082[-0.045$, $0.208]$ for PLG and $B=0.014$ [ $-0.016,0.043]$ for HbA1c. The same was true for dietary ALA intake $(B=0.249$ $[-0.275,0.773]$ for FPG, $B=0.937[-0.520,2.40]$ for PLG and $B=0.196[-0.131,0.524]$ for $\mathrm{HbA} 1 \mathrm{c})$.

\section{Discussion and conclusion}

In the present population-based cross-sectional study, we observed significant inverse associations of LA in serum with concentrations of FPG and PLG, but not with HbA1c. The proportion of ALA in serum was not associated with these markers of glucose metabolism. These associations were independent of age, sex, total energy intake, obesity, and other lifestyle factors. In prospective analyses of this same population, however, there were no significant independent associations between serum LA and ALA proportions and blood glucose concentrations. 


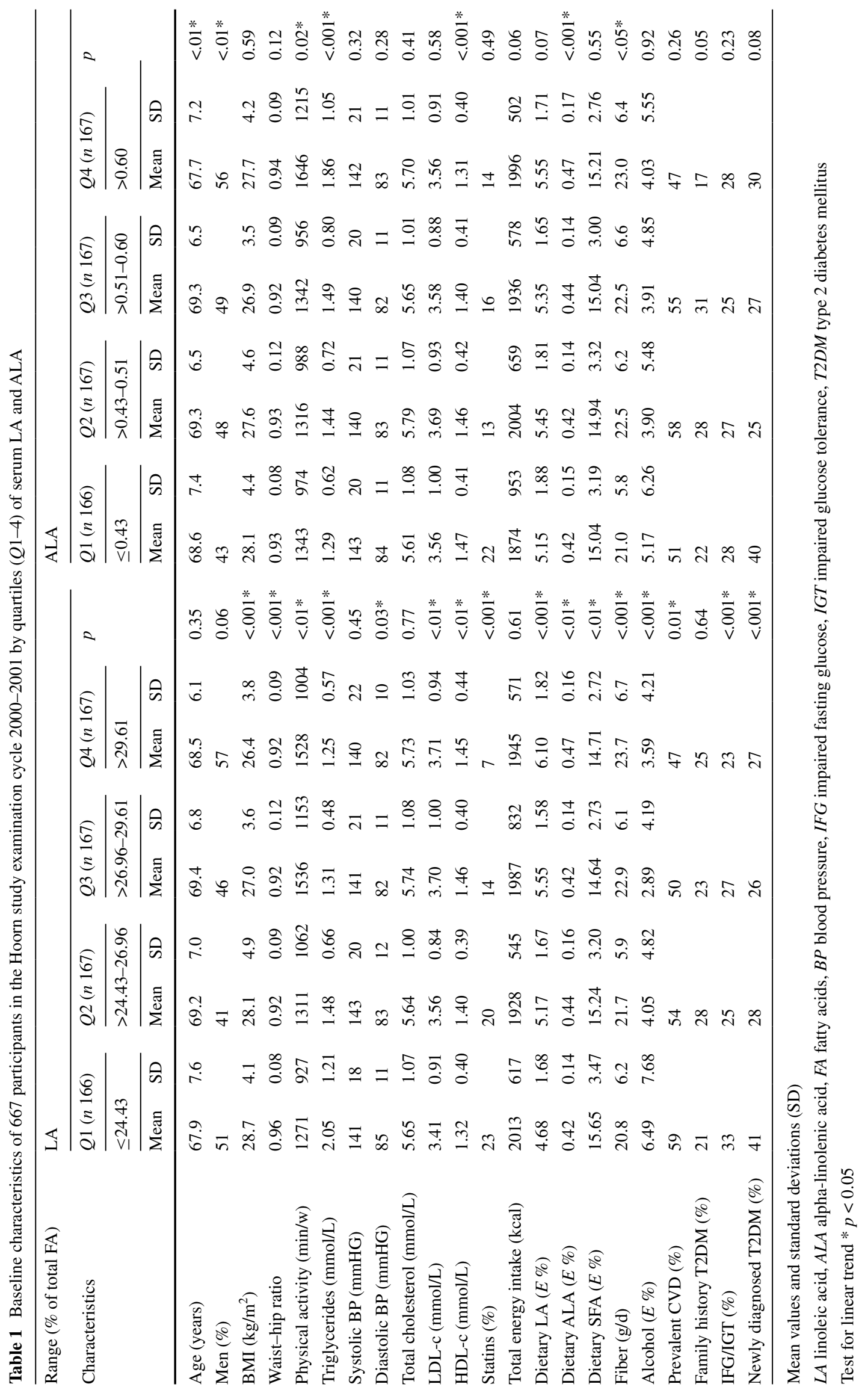


Table 2 Unstandardized regression coefficients of a cross-sectional linear regression analysis testing the association of serum LA and ALA proportions with three blood glucose parameters

\begin{tabular}{|c|c|c|c|}
\hline & FPG mmol/L $(n=667)$ & PLG mmol/L $(n=537)$ & $\operatorname{HbA1c}(\%)(n=664)$ \\
\hline & $B[95 \% \mathrm{CI}]$ & $B[95 \% \mathrm{CI}]$ & $B[95 \% \mathrm{CI}]$ \\
\hline \multicolumn{4}{|l|}{ LA $(\%)$} \\
\hline Model I & $-0.054[-0.077,-0.031]^{*}$ & $-0.130[-0.186,-0.074]^{*}$ & $-0.006[-0.019,0.006]$ \\
\hline Model II & $-0.027[-0.050,-0.004]^{*}$ & $-0.091[-0.148,-0.034]^{*}$ & $0.004[-0.009,0.017]$ \\
\hline Model III & $-0.024[-0.045,-0.002]^{*}$ & $-0.099[-0.158,-0.039]^{*}$ & $0.000[-0.014,0.013]$ \\
\hline+ Cholesterol and statins ${ }^{\mathrm{a}}$ & $-0.021[-0.044,0.001]$ & $-0.096[-0.156,-0.035]^{*}$ & $0.003[-0.011,0.017]$ \\
\hline+ Triglycerides $^{b}$ & $-0.004[-0.027,0.019]$ & $-0.054[-0.117,0.009]$ & $0.012[-0.003,0.026]$ \\
\hline \multicolumn{4}{|l|}{$\operatorname{ALA}(\%)$} \\
\hline Model I & $-0.534[-1.111,0.043]$ & $-1.192[-2.680,0.296]$ & $-0.076[-0.395,0.244]$ \\
\hline Model II & $-0.508[-1.056,0.041]$ & $-1.291[-2.737,0.154]$ & $-0.088[-0.404,0.228]$ \\
\hline Model III & $-0.292[-0.794,0.210]$ & $-0.978[-2.439,0.482]$ & $-0.057[-0.372,0.258]$ \\
\hline+ Cholesterol and statins ${ }^{\mathrm{a}}$ & $-0.256[-0.759,0.247]$ & $-0.894[-2.366,0.578]$ & $-0.046[-0.361,0.268]$ \\
\hline+ Triglycerides $^{\mathrm{b}}$ & $-0.810[-1.333,-0.288]^{*}$ & $-2.335[-3.837,-0.834]^{*}$ & $-0.319[-0.650,0.012]$ \\
\hline
\end{tabular}

Model I is adjusted for age, sex, and total energy intake

Model II is adjusted for age, sex, total energy intake, BMI, and WHR

Model III is adjusted for age, sex, total energy intake, BMI, WHR, physical activity, fiber, dietary SFA, alcohol, and education level

$F P G$ fasting plasma glucose, $P L G$ post-load glucose, $H b A 1 c$ glycated hemoglobin, $B$ unstandardized regression coefficient, $L A$ linoleic acid, $A L A$ alpha-linolenic acid

Linear regression analyses with $* p<0.05$

a Stepwise adjustments (not cumulative) for cholesterol and statins to model III

b Stepwise adjustments (not cumulative) for triglycerides to model III

\section{Strengths and limitations}

A major strength of this study is the measurement of the proportions of LA and ALA in serum as markers of their dietary intake. Intake of fatty acids can be difficult to estimate accurately from FFQ because of misreporting by subjects and lack of data on fatty acid content in food composition tables [7]. As a result, important associations between fatty acid intake and health outcomes can be missed when only assessed with dietary fat intake from a FFQ [18]. This is supported by the current study, in which we found an inverse cross-sectional association between serum LA and glucose concentration, while this was not observed for dietary LA.

Total fatty acid levels in serum, as measured in the present study, originate from different blood lipid fractions including triglycerides, cholesteryl esters, phospholipids, and nonesterified fatty acids. These lipid fractions reflect dietary intake in previous days to weeks at maximum [18]. Short-term dietary modifications reflected in serum fatty acids may not translate into changes in risk factors within the same timeframe, thereby potentially underestimating the true association. Nevertheless, serum LA and ALA levels do reasonably well correlate with dietary LA and ALA intake, as assessed via FFQ (typically reflecting intake over previous 4 weeks) [18].

An advantage of the current study is that we determined FPG, PLG, as well as HbA1c, three markers of different aspects of glucose metabolism, that are each considered relevant and are accepted for the diagnosis of T2DM [32]. This provides the opportunity to investigate the associations of serum and dietary LA and ALA with different aspects of the glucose metabolism. Other strengths of the current study include the population-based prospective design and the detailed characteristics of the study population.

Several limitations of our analysis are to be addressed. It might be argued that the overrepresentation of T2DM patients and persons with impaired glucose tolerance in our population limits the generalizability of our findings to other populations. On the other hand, the advantage of a population with highly prevalent T2DM and impaired glucose tolerance is that this enhances the likelihood to detect larger differences in glucose metabolism between subjects. Regardless, the current study and in particular the prospective analyses are limited by sample size and may thus lack power to assess a potential association with diabetes incidence. Serum fatty acid proportions and glucose parameters can be influenced by age, sex, total energy intake, obesity, lifestyle factors, and other (unknown) confounders. Despite careful adjustments for most of these variables, we cannot rule out residual or unmeasured confounding. There is also the chance that our results were over-adjusted by including waist-hip ratio as potential confounder in the model. There are indications that PUFA, in comparison with SFA, may reduce abdominal fat 
Table 3 Unstandardized regression coefficients of a prospective linear regression analysis testing the association of LA and ALA serum proportions at baseline with three blood glucose parameters at follow-up

\begin{tabular}{|c|c|c|c|}
\hline & FPG mmol/L $(n=257)$ & PLG mmol/L $(n=253)$ & $\operatorname{HbA1c}(\%)(n=257)$ \\
\hline & $B[95 \% \mathrm{CI}]$ & $B[95 \% \mathrm{CI}]$ & $B[95 \% \mathrm{CI}]$ \\
\hline \multicolumn{4}{|l|}{ LA $(\%)$} \\
\hline Model I & $-0.025[-0.050,0.000]$ & $-0.112[-0.187,-0.037]^{*}$ & $-0.012[-0.025,0.001]$ \\
\hline Model II & $-0.005[-0.025,0.016]$ & $-0.054[-0.123,0.015]$ & $-0.010[-0.021,0.001]$ \\
\hline Model III & $0.003[-0.019,0.025]$ & $-0.026[-0.100,0.049]$ & $-0.007[-0.019,0.005]$ \\
\hline+ Cholesterol and statins $^{\mathrm{a}}$ & $0.004[-0.019,0.026]$ & $-0.045[-0.124,0.033]$ & $-0.002[-0.015,0.010]$ \\
\hline+ Triglyceride $^{\mathrm{b}}$ & $0.004[-0.019,0.026]$ & $-0.045[-0.124,0.033]$ & $-0.002[-0.015,0.010]$ \\
\hline \multicolumn{4}{|l|}{$\operatorname{ALA}(\%)$} \\
\hline Model I & $-0.009[-0.601,0.584]$ & $-1.616[-3.426,0.195]$ & $0.043[-0.260,0.346]$ \\
\hline Model II & $0.107[-0.371,0.585]$ & $-1.240[-2.853,0.372]$ & $0.065[-0.198,0.327]$ \\
\hline Model III & $0.107[-0.374,0.588]$ & $-1.098[-2.716,0.521]$ & $0.072[-0.191,0.336]$ \\
\hline+ Cholesterol and statins $^{\mathrm{a}}$ & $0.114[-0.375,0.603]$ & $-1.044[-2.765,0.677]$ & $0.128[-0.152,0.408]$ \\
\hline+ Triglycerides $^{\mathrm{b}}$ & $0.114[-0.375,0.603]$ & $-1.044[-2.765,0.677]$ & $0.128[-0.152,0.408]$ \\
\hline
\end{tabular}

Model I is adjusted for age, sex, and total energy intake

Model II is adjusted for age, sex, total energy intake, and baseline glycemic measure

Model III is adjusted for age, sex, total energy intake, baseline glycemic measure, BMI, WHR, physical activity, fiber, dietary SFA, alcohol, and education level

$F P G$ fasting plasma glucose, $P L G$ post-load glucose, $H b A l c$ glycated hemoglobin, $B$ unstandardized regression coefficient, $L A$ linoleic acid, $A L A$ alpha-linolenic acid

Linear regression analyses with $* p<0.05$

${ }^{\text {a }}$ Stepwise adjustments (not cumulative) for cholesterol and statins to model III

b Stepwise adjustments (not cumulative) for triglycerides to model III

[37], which could lead to a reduction in blood glucose. The waist-hip ratio reflecting abdominal fat could thus be in the causal pathway from dietary and serum LA to T2DM, in which case the adjustment for waist-hip ratio would underestimate their relationship in the population. Another study limitation is that the proportions of LA and ALA in serum were assessed only once at baseline. Potential changes in intake of LA and ALA over time could have caused misclassification of long-term dietary exposure and in this way underestimation of the underlying association.

\section{Current findings in the context of other studies}

As in most epidemiological studies, serum fatty acid levels in the current study were measured and expressed as proportion of total fatty acids. We observed that the correlation of dietary LA and ALA intake with serum proportions of LA and ALA is stronger than the correlation with concentrations of LA and ALA (present study). This may favor the expression of LA and ALA as a proportion of total fatty acids.

We found that higher proportions of serum LA in cross-sectional analyses were associated with lower levels of blood glucose concentrations. These findings are in line with results from earlier prospective cohort studies on the relationship between serum LA and T2DM incidence
$[13,14,19-23]$. Our findings are also largely in line with results from a recent systematic review, which concluded that higher serum levels of ALA tend to be associated with lower risk of T2DM [24]. LA or ALA proportions in serum did not show an association with HbA1c. This may suggest that for HbA1c physiological and pathological determinants other than diet are more important than for glucose [29].

There was a weak correlation between serum ALA and markers of blood glucose metabolism in our study, which has also been observed in other studies [38]. It has been suggested to be the result of rapid oxidation of dietary ALA in the body [39] or of measurement error in the intake data including underlying food composition tables [38].

\section{Potential mechanisms}

Biological mechanisms underlying a possible relation between dietary and serum fatty acids and risk of T2DM are not completely understood. It has been hypothesized that consuming a diet with a high ratio of PUFA to SFA increases the PUFA content of phospholipids, which may improve the glucose transport, oxidation, and lipogenesis [40]. The ratio of PUFA to SFA may influence the flexibility of the cell membrane, cellular function, and insulin signaling leading to an increase in insulin sensitivity 
when more PUFA is consumed. Indeed, one intervention trial in which PUFA was replaced by SFA reported a beneficial effect on insulin sensitivity [41]. In the present study, serum LA proportions showed a significant inverse association with both FPG and PLG. This is plausible given that FPG and PLG are the primary markers of insulin sensitivity in the liver and the peripheral tissues, respectively [42]. Part of the observed association between LA proportions and T2DM risk in our study was explained by differences in triglyceride levels. Dyslipidemia, involving elevated triglyceride levels, is a common characteristic in patients with T2DM [43]. In addition, since the proportion of LA varies between lipid species, the lipid levels may have confounded the relationship between LA and glucose. In cholesteryl esters, LA compose over half of fatty acids, whereas in triglycerides the proportion of LA is about $15 \%$ and in phospholipids about $20 \%$. This implies that among individuals with higher concentrations of triglycerides, the proportion of LA in total serum is lower [18]. However, the causality of the relationship between LA proportion and lipid species is poorly understood. One might suggest that increases in triglycerides lead to a reduction in LA levels. On the other hand, changes in triglycerides, and its associated etiologies insulin resistance and ectopic fat deposition may be the result of dietary LA intake. The latter is supported by controlled human dietary intervention studies, in which PUFA as replacement of SFA reduced liver fat $[44,37]$ and insulin resistance [41]. In addition, a meta-analysis of 60 controlled intervention studies on blood lipids has established that dietary PUFA intake reduce triglyceride levels compared with carbohydrates [1]. Thus, adjustment for triglycerides in our models will correct for variation in LA between lipid species, but may lead to underestimation of the true association because it partially corrects for triglycerides as an intermediate in the causal pathway toward higher glucose levels. Therefore, we assume that true association between LA and glucose levels may lie in between the associations estimated by the triglycerideadjusted and unadjusted models.

The proportion of LA in serum fatty acid is not solely influenced by LA intake, but also by endogenous fatty acid metabolism [38]. For example, an increased $\Delta 6$-desaturase activity, resulting in lower serum LA, has been associated with insulin resistance and T2DM [45, 46]. Therefore, reversed causality cannot be excluded as an explanation for the observed association. For the present study, however, this is not very likely given that $\Delta 6$-desaturase activity (estimated as C18:3n6/C18:2n6 ratio) in T2DM subjects was not significantly different from nondiabetic subjects (data not shown). The biological mechanisms by which different types of PUFAs might affect glucose metabolism remain to be elucidated.

\section{Conclusion}

In this population with overrepresentation of subjects with glucose intolerance, serum LA was inversely associated with fasting and post-load glucose in cross-sectional, but not in prospective analyses. Further studies are needed to elucidate the potential role of serum LA and ALA levels and dietary PUFA in glucose metabolism.

Acknowledgments Research Council of Norway funded the measurements of fatty acids levels. The Hoorn study has been supported by grants from the Netherlands Organization for Scientific Research (NWO), the Dutch Diabetes Research Foundation, the Netherlands Heart Foundation, and Novartis Pharma BV, the Netherlands.

Authors' contribution The authors' contributions were as follows: JMD, GN, and CDAS were the principal investigators of the Hoorn study and designed the present study with HR, IAB, and MA. MC wrote the manuscript and analyzed and interpreted the data with MA, IAB, JMD, and PLZ. JMD, GN, and AE coordinated and supervised the data collection. All authors reviewed the manuscript and provided comments. All authors approved the final manuscript.

Funding Research Council of Norway; Netherlands Organization for Scientific Research (NWO); the Dutch Diabetes Research Foundation; the Netherlands Heart Foundation; Novartis Pharma BV, the Netherlands.

\section{Compliance with ethical standards}

Conflict of interest MA and PZ are employees of Unilever. MC was a Unilever employee at the time of conducting the study. Unilever markets food products made of vegetable oils, including margarines and dressings. Other authors have no conflicts of interest to declare.

Ethical standard All human studies have been approved by the VU University Medical Centre Ethics Committee and have therefore been performed in accordance with the ethical standards laid down in the 1964 Declaration of Helsinki and its later amendments. All persons gave their informed consent prior to their inclusion in the study.

Open Access This article is distributed under the terms of the Creative Commons Attribution 4.0 International License (http://creativecommons.org/licenses/by/4.0/), which permits unrestricted use, distribution, and reproduction in any medium, provided you give appropriate credit to the original author(s) and the source, provide a link to the Creative Commons license, and indicate if changes were made.

\section{References}

1. Mensink RP, Zock PL, Kester AD, Katan MB (2003) Effects of dietary fatty acids and carbohydrates on the ratio of serum total to HDL cholesterol and on serum lipids and apolipoproteins: a meta-analysis of 60 controlled trials. Am J Clin Nutr 77:1146-1155

2. Farvid MS, Ding M, Pan A, Sun Q, Chiuve SE, Steffen LM, Willett WC, Hu FB (2014) Dietary linoleic acid and risk of coronary heart disease: a systematic review and meta-analysis of prospective cohort studies. Circulation 130:1568-1578 
3. The Joint FAO/WHO Expert Consultation on Fats and Fatty Acids in Human Nutrition (2010) Interim Summary of Conclusion and Dietary Recommendations on Total Fat and Fatty Acids. WHO HQ, Geneva http://www.who.int/nutrition/topics/FFA_ summary_rec_conclusion.pdf

4. Perk J, De BG, Gohlke H, Graham I, Reiner Z, Verschuren M, Albus C, Benlian P, Boysen G, Cifkova R, Deaton C, Ebrahim S, Fisher M, Germano G, Hobbs R, Hoes A, Karadeniz S, Mezzani A, Prescott E, Ryden L, Scherer M, Syvanne M, Scholte op Reimer WJ, Vrints C, Wood D, Zamorano JL, Zannad F (2012) European guidelines on cardiovascular disease prevention in clinical practice (version 2012). The Fifth Joint Task Force of the European Society of Cardiology and Other Societies on Cardiovascular Disease Prevention in Clinical Practice (constituted by representatives of nine societies and by invited experts). Eur Heart J 33:1635-1701

5. Schwab U, Lauritzen L, Tholstrup T, Haldorssoni T, Riserus U, Uusitupa M, Becker W (2014) Effect of the amount and type of dietary fat on cardiometabolic risk factors and risk of developing type 2 diabetes, cardiovascular diseases, and cancer: a systematic review. Food Nutr Res 58:25145. doi:10.3402/fnr.v58.25145

6. International Diabetes Federation (2015) IDF diabetes Atlas, 7th edn. International Diabetes Federation, Brussels, Belgium http:// www.idf.org/diabetesatlas Accessed 8 Nov 2015

7. Food and Agriculture Organization of the United Nations. FAO report of an expert consultation on fats and fatty acids in human nutrition. ISSN 02544725 FAO Food and Nutrition Paper 91. 2010. Rome, Italy 2010

8. Meyer KA, Kushi LH, Jacobs DR Jr, Folsom AR (2001) Dietary fat and incidence of type 2 diabetes in older Iowa women. Diabetes Care 24:1528-1535

9. Salmeron J, Hu FB, Manson JE, Stampfer MJ, Colditz GA, Rimm EB, Willett WC (2001) Dietary fat intake and risk of type 2 diabetes in women. Am J Clin Nutr 73:1019-1026

10. Harding AH, Day NE, Khaw KT, Bingham S, Luben R, Welsh A, Wareham NJ (2004) Dietary fat and the risk of clinical type 2 diabetes: the European prospective investigation of Cancer-Norfolk study. Am J Epidemiol 159:73-82

11. Patel PS, Sharp SJ, Jansen E, Luben RN, Khaw KT, Wareham NJ, Forouhi NG (2010) Fatty acids measured in plasma and erythrocyte-membrane phospholipids and derived by food-frequency questionnaire and the risk of new-onset type 2 diabetes: a pilot study in the European Prospective Investigation into Cancer and Nutrition (EPIC)-Norfolk cohort. Am J Clin Nutr 92:1214-1222

12. van Dam RM, Willett WC, Rimm EB, Stampfer MJ, Hu FB (2002) Dietary fat and meat intake in relation to risk of type 2 diabetes in men. Diabetes Care 25:417-424

13. Kroger J, Zietemann V, Enzenbach C, Weikert C, Jansen EH, Doring F, Joost HG, Boeing H, Schulze MB (2011) Erythrocyte membrane phospholipid fatty acids, desaturase activity, and dietary fatty acids in relation to risk of type 2 diabetes in the European Prospective Investigation into Cancer and Nutrition (EPIC)Potsdam Study. Am J Clin Nutr 93:127-142

14. Hodge AM, English DR, O'Dea K, Sinclair AJ, Makrides M, Gibson RA, Giles GG (2007) Plasma phospholipid and dietary fatty acids as predictors of type 2 diabetes: interpreting the role of linoleic acid. Am J Clin Nutr 86:189-197

15. Voss S, Kroke A, Klipstein-Grobusch K, Boeing H (1998) Is macronutrient composition of dietary intake data affected by underreporting? Results from the EPIC-Potsdam Study. European Prospective Investigation into Cancer and Nutrition. Eur J Clin Nutr 52:119-126

16. Goris AH, Westerterp-Plantenga MS, Westerterp KR (2000) Undereating and underrecording of habitual food intake in obese men: selective underreporting of fat intake. Am J Clin Nutr 71:130-134

17. Ma J, Folsom AR, Shahar E, Eckfeldt JH (1995) Plasma fatty acid composition as an indicator of habitual dietary fat intake in middle-aged adults. The Atherosclerosis Risk in Communities (ARIC) Study Investigators. Am J Clin Nutr 62:564-571

18. Hodson L, Skeaff CM, Fielding BA (2008) Fatty acid composition of adipose tissue and blood in humans and its use as a biomarker of dietary intake. Prog Lipid Res 47:348-380

19. Lankinen MA, Stancakova A, Uusitupa M, Agren J, Pihlajamaki J, Kuusisto J, Schwab U, Laakso M (2015) Plasma fatty acids as predictors of glycaemia and type 2 diabetes. Diabetologia 58:2533-2544

20. Vessby B, Aro A, Skarfors E, Berglund L, Salminen I, Lithell $\mathrm{H}$ (1994) The risk to develop NIDDM is related to the fatty acid composition of the serum cholesterol esters. Diabetes 43:1353-1357

21. Wang L, Folsom AR, Zheng ZJ, Pankow JS, Eckfeldt JH (2003) Plasma fatty acid composition and incidence of diabetes in middle-aged adults: the Atherosclerosis Risk in Communities (ARIC) Study. Am J Clin Nutr 78:91-98

22. Mahendran Y, Agren J, Uusitupa M, Cederberg H, Vangipurapu J, Stancakova A, Schwab U, Kuusisto J, Laakso M (2014) Association of erythrocyte membrane fatty acids with changes in glycemia and risk of type 2 diabetes. Am J Clin Nutr 99:79-85

23. Krachler B, Norberg M, Eriksson JW, Hallmans G, Johansson I, Vessby B, Weinehall L, Lindahl B (2008) Fatty acid profile of the erythrocyte membrane preceding development of type 2 diabetes mellitus. Nutr Metab Cardiovasc Dis 18:503-510

24. Wu JH, Micha R, Imamura F, Pan A, Biggs ML, Ajaz O, Djousse L, Hu FB, Mozaffarian D (2012) Omega-3 fatty acids and incident type 2 diabetes: a systematic review and meta-analysis. Br J Nutr 107(Suppl 2):S214-S227

25. Mooy JM, Grootenhuis PA, de Vries H, Valkenburg HA, Bouter LM, Kostense PJ, Heine RJ (1995) Prevalence and determinants of glucose intolerance in a Dutch Caucasian population. Hoorn stud. Diabetes Care 18:1270-1273

26. Bos G, Poortvliet MC, Scheffer PG, Dekker JM, Ocke MC, Nijpels G, Stehouwer CD, Bouter LM, Teerlink T, Heine RJ (2007) Dietary polyunsaturated fat intake is associated with lowdensity lipoprotein size, but not with susceptibility to oxidation in subjects with impaired glucose metabolism and type II diabetes: the Hoorn study. Eur J Clin Nutr 61:205-211

27. Spijkerman AM, Dekker JM, Nijpels G, Adriaanse MC, Kostense PJ, Ruwaard D, Stehouwer CD, Bouter LM, Heine RJ (2003) Microvascular complications at time of diagnosis of type 2 diabetes are similar among diabetic patients detected by targeted screening and patients newly diagnosed in general practice: the Hoorn screening study. Diabetes Care 26:2604-2608

28. van den Hurk K, Alssema M, Kamp O, Henry RM, Stehouwer CD, Smulders YM, Nijpels G, Paulus WJ, Dekker JM (2012) Independent associations of glucose status and arterial stiffness with left ventricular diastolic dysfunction: an 8-year follow-up of the Hoorn study. Diabetes Care 35:1258-1264

29. Lapolla A, Mosca A, Fedele D (2011) The general use of glycated haemoglobin for the diagnosis of diabetes and other categories of glucose intolerance: still a long way to go. Nutr Metab Cardiovasc Dis 21:467-475

30. van den Berg E, Biessels GJ, Stehouwer CD, Kappelle LJ, Heine RJ, Nijpels G, Dekker JM (2010) Ten-year time course of risk factors for increased carotid intima-media thickness: the Hoorn Study. Eur J Cardiovasc Prev Rehabil 17:168-174

31. World Health Organization (2011) Use of glycated haemoglobin $(\mathrm{HbA} 1 \mathrm{c})$ in the diagnosis of diabetes mellitus. WHO/NMH/ CHP/CPM/11.1. Geneva, Switzerland http://www.who.int/ 
diabetes/publications/report-hba1c_2011.pdf?ua=1 Accessed 10 Aug 2015

32. World Health Organization (2006) Definition and diagnosis of diabetes mellitus and intermediate hyperglycemia: report of a WHO/IDF consultation. NLM classification: WK 810. Geneva, Switzerland http://apps.who.int/iris/bitstream/10665/43588/1/9241594934_eng.pdf Accessed 9 Aug 2015

33. Henry RM, Kostense PJ, Spijkerman AM, Dekker JM, Nijpels G, Heine RJ, Kamp O, Westerhof N, Bouter LM, Stehouwer CD (2003) Arterial stiffness increases with deteriorating glucose tolerance status: the Hoorn study. Circulation 107:2089-2095

34. Wendel-Vos GC, Schuit AJ, Saris WH, Kromhout D (2003) Reproducibility and relative validity of the short questionnaire to assess health-enhancing physical activity. J Clin Epidemiol 56:1163-1169

35. Grootenhuis PA, Westenbrink S, Sie CM, de Neeling JN, Kok FJ, Bouter LM (1995) A semiquantitative food frequency questionnaire for use in epidemiologic research among the elderly: validation by comparison with dietary history. J Clin Epidemiol 48:859-868

36. Voedingscentrum DHTN (2001) Dutch food composition database: NEVO. Bilthoven, The Netherlands

37. Rosqvist F, Iggman D, Kullberg J, Cedernaes J, Johansson HE, Larsson A, Johansson L, Ahlstrom H, Arner P, Dahlman I, Riserus $U$ (2014) Overfeeding polyunsaturated and saturated fat causes distinct effects on liver and visceral fat accumulation in humans. Diabetes 63:2356-2368

38. Pan A, Chen M, Chowdhury R, Wu JH, Sun Q, Campos H, Mozaffarian D, Hu FB (2012) Alpha-linolenic acid and risk of cardiovascular disease: a systematic review and meta-analysis. Am J Clin Nutr 96:1262-1273
39. Arterburn LM, Hall EB, Oken H (2006) Distribution, interconversion, and dose response of $n-3$ fatty acids in humans. Am J Clin Nutr 83:1467S-1476S

40. Field CJ, Ryan EA, Thomson AB, Clandinin MT (1990) Diet fat composition alters membrane phospholipid composition, insulin binding, and glucose metabolism in adipocytes from control and diabetic animals. J Biol Chem 265:11143-11150

41. Summers LK, Fielding BA, Bradshaw HA, Ilic V, Beysen C, Clark ML, Moore NR, Frayn KN (2002) Substituting dietary saturated fat with polyunsaturated fat changes abdominal fat distribution and improves insulin sensitivity. Diabetologia 45:369-377

42. DeFronzo RA, Tripathy D (2009) Skeletal muscle insulin resistance is the primary defect in type 2 diabetes. Diabetes Care 32(Suppl 2):S157-S163

43. Taskinen MR (2003) LDL-cholesterol, HDL-cholesterol or triglycerides-Which is the culprit? Diabetes Res Clin Pract 61(Suppl 1):S19-S26

44. Bjermo H, Iggman D, Kullberg J, Dahlman I, Johansson L, Persson L, Berglund J, Pulkki K, Basu S, Uusitupa M, Rudling M, Arner P, Cederholm T, Ahlstrom H, Riserus U (2012) Effects of n-6 PUFAs compared with SFAs on liver fat, lipoproteins, and inflammation in abdominal obesity: a randomized controlled trial. Am J Clin Nutr 95:1003-1012

45. Nakamura MT, Nara TY (2004) Structure, function, and dietary regulation of delta6, delta5, and delta9 desaturases. Annu Rev Nutr 24:345-376

46. Warensjo E, Riserus U, Vessby B (2005) Fatty acid composition of serum lipids predicts the development of the metabolic syndrome in men. Diabetologia 48:1999-2005 